\title{
Frontières
}

\section{Le fardeau économique de la douleur et de son soulagement}

\section{Jean Lachaine}

Volume 17, numéro 2, printemps 2005

Surtout, ne pas souffrir

URI : https://id.erudit.org/iderudit/1073495ar

DOI : https://doi.org/10.7202/1073495ar

Aller au sommaire du numéro

Éditeur(s)

Université du Québec à Montréal

ISSN

1180-3479 (imprimé)

1916-0976 (numérique)

Découvrir la revue

Citer ce document

Lachaine, J. (2005). Le fardeau économique de la douleur et de son

soulagement. Frontières, 17(2), 89-91. https://doi.org/10.7202/1073495ar d'utilisation que vous pouvez consulter en ligne.

https://apropos.erudit.org/fr/usagers/politique-dutilisation/ 


\section{Le fardeau économique de la douleur et de son soulagement}

\section{Jean Lachaine, Ph. D., Faculté de pharmacie, Université de Montréal.}

La douleur représente un problème de santé majeur. Elle affecte, à des degrés divers, une proportion importante de la population. En plus d'avoir un impact considérable sur la qualité de vie des patients qui en souffrent, elle représente aussi pour nos systèmes de soins de santé et pour la société en général un fardeau économique substantiel.

Dans le contexte qui prévaut actuellement, où les ressources pouvant être allouées à la santé sont limitées, il est essentiel de se préoccuper des aspects économiques liés à la santé. En effet, les attentes des populations en matière de soins de santé sont pratiquement illimitées, mais force est de constater que nos sociétés n'ont pas nécessairement la capacité de payer pour toutes les interventions de santé disponibles. Ainsi, la douleur autant que tout autre problème de santé se doit d'être prise en compte en considérant aussi ses dimensions économiques.

Limpact économique associé à la douleur est de différents ordres. D'une part, la douleur engendre des coûts directs de santé qui comprennent, entre autres, les coûts liés aux consultations médicales, aux visites à l'urgence et le cas échéant aux hospitalisations, le coût des médicaments utilisés pour le soulagement de la douleur, les coûts liés aux examens et tests diagnostiques tels que les radiographies ou les tests de laboratoire. La douleur représente d'ailleurs une des principales raisons de visite chez le médecin (Katz, 2002). La douleur entraîne aussi d'autres coûts tels que ceux associés aux consultations de chiropraticiens, ostéopathes ou massothérapeutes ainsi que ceux associés aux approches de la médecine alternative, l'homéopathie par exemple.

En outre, selon une perspective de la société, la douleur entraîne des conséquences économiques considérables liées à la perte de productivité des personnes qui en sont affligés. À ce propos, une étude américaine récente effectuée auprès d'un échantillon aléatoire de près de 29000 travailleurs a permis d'estimer la proportion de travailleurs qui avaient subi une perte de productivité liée à la douleur au cours des deux semaines précédant l'enquête (Stewart et al., 2003). On a trouvé dans cette étude que $13 \%$ des travailleurs ont eu une telle perte de productivité. Les causes de douleur rapportées le plus fréquemment ont été les céphalées, suivi des douleurs lombaires, douleurs arthritiques et douleurs musculosquelettiques. Une perte de productivité moyenne de 4,6 heures par semaine était par ailleurs rapportée. On a estimé que cette perte de productivité, pour l'ensemble de la population des États-Unis, correspondait à un coût de 61,2 milliards de dollars par année ${ }^{1}$. Il ressort aussi de cette étude que la majorité de ces coûts ne sont pas liés à l'absence au travail, mais sont en plus grande partie dus à une performance réduite à cause de la douleur.

La douleur représente une problématique fort complexe qui peut d'ailleurs se présenter sous de nombreuses facettes: elle peut être chronique ou aiguë, elle peut avoir différents degrés d'intensité, elle peut être associée à différentes maladies, et par ailleurs, elle peut être perçue de façon très différente d'une personne à l'autre. Ainsi la douleur est un problème de santé difficile à circonscrire et par conséquent il est difficile d'évaluer avec justesse les diverses conséquences qu'elle peut entraîner, notamment les conséquences économiques.

L'ampleur du fardeau économique de la douleur est en partie liée au grand nombre de personnes qui souffrent. En effet, bien qu'il soit difficile d'en faire une estimation précise, la proportion de la population qui ressent de la douleur est considérable. La douleur chronique est d'ailleurs la principale cause d'invalidité (Phillips, 2001). En outre, les conséquences économiques liées à la douleur dépendent de l'importance de l'approche thérapeutique qui sera déployée pour tenter de la contrôler. On constate aussi que l'ampleur des coûts liés à la douleur varie selon la méthode utilisée pour les estimés. C'est particulièrement le cas lorsqu'on tente d'estimer les coûts associés à la perte de productivité. Les différentes méthodes proposées pour évaluer de tels coûts fournissent des estimés qui peuvent être très différents (Hutubessy et al., 1999; Rothermich et Pathak, 1999; Koopmanschap et al., 1995).

Il est difficile de tenter de quantifier l'impact économique de la douleur de façon globale. D'ailleurs, les études qui ont tenté d'évaluer l'impact économique de la douleur se sont limitées à des douleurs bien spécifiques. On retrouve entre autres des études sur les dimensions économiques de la douleur dans le cas des douleurs lombaires, des douleurs neuropathiques, des douleurs en oncologie et des douleurs post-chirurgicales. Évidemment, il y a plusieurs études portant sur les aspects économiques de maladies ayant une composante 


\section{d'un coup je cherche asile peu importe où...}

«douleur» importante, comme l'arthrose par exemple, mais les évaluations économiques portant sur ces maladies ne sont généralement pas spécifiques à la douleur mais portent sur la maladie dans son ensemble.

Parmi les différents types de douleur, la douleur lombaire est celle pour laquelle l'impact économique a été le plus étudié et discuté. Cela peut s'expliquer par le nombre de personnes qui en souffrent et l'importance des conséquences économiques qui lui sont associées. Les coûts associés aux douleurs lombaires seraient comparables à ceux d'autres maladies largement répandues telles que les maladies cardiaques, la dépression ou le diabète (Maetzel et Li, 2002). De plus, la douleur lombaire est une cause courante d'interventions chirurgicales et représente un motif fréquent d'hospitalisation (Pai et Sundaram, 2004). Après la grippe, la douleur lombaire est la deuxième cause d'absence au travail, représentant environ $40 \%$ de toutes les absences dues à un problème de santé (Wildenhaus, 2004). Dans une étude visant à estimer les dépenses de santé (services médicaux et médication) des patients atteints de douleurs chroniques lombaires par rapport à celles d'un groupe de sujets comparables selon l'âge et le sexe mais n'ayant pas de douleurs lombaires, on a trouvé que les coûts de santé globaux des patients avec douleurs lombaires étaient plus du double de ceux des patients non affligés par ce problème (4349 \$US contre 1643 \$US) (Mapel et al., 2004). Dans une autre étude américaine le coût annuel par personne associé aux douleurs d'origine vertébrale a été estimé entre 9000 \$US et 19000 \$US (Straus, 2002). Par ailleurs, selon la perspective de l'employeur, on a estimé que le coût annuel associé à la perte de productivité due aux douleurs lombaires serait de 7806 \$US par employé (Joish et Brixner, 2002).

La douleur neuropathique constitue aussi une forme de douleur relativement répandue. Dans une étude comparant des sujets atteints de douleurs neuropathiques à des sujets de même âge et sexe mais ne souffrant pas de telles douleurs, on a trouvé que les coûts de santé des patients atteints de douleurs neuropathiques étaient trois fois plus élevés que ceux du groupe témoin (Berger et al., 2004).

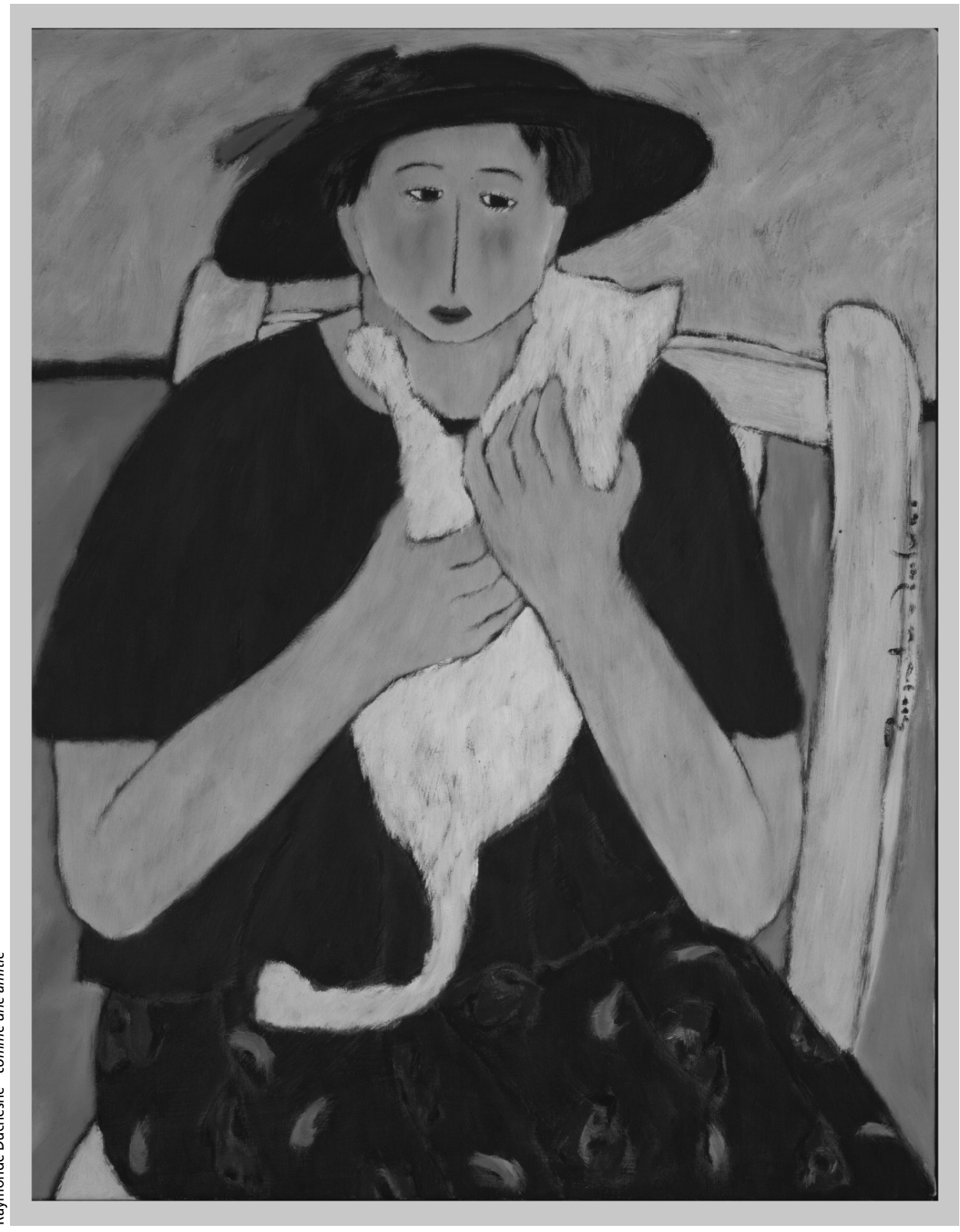

Le cancer est aussi une maladie où la douleur est très souvent présente, soit par la maladie elle-même selon la localisation de la tumeur, soit par l'effet de la tumeur sur d'autres fonctions de l'organisme ou par les interventions visant à traiter le cancer. À ce chapitre, on peut citer la chirurgie, la radiothérapie et la chimiothérapie qui toutes trois peuvent entraîner de la douleur. Il a été estimé que 40\% des patients ayant un cancer localisé et $70 \%$ de ceux ayant un cancer disséminé souffriront de douleurs (Abernethy et al., 2003). En utilisant la méthode de la propension à payer, des chercheurs ont étudié la valeur monétaire associée à la douleur dans le cancer de la prostate. Ils ont estimé que la valeur économique de cette douleur correspondait à 16600 Euros par personne atteinte (Sennfalt et al., 2004).

Bien que les coûts liés à la perte de productivité soient les plus importants dans l'ensemble des coûts engendrés par la douleur (Maniadakis et Gray, 2000), les médicaments utilisés pour le soulagement de la douleur et le recours aux services médicaux représentent une dépense non négligeable. Au Québec, en 2003, plus d'un million de personnes, soit près de la moitié des participants au régime d'assurance médicaments de la Régie de l'assurance maladie du Québec (RAMQ), ont reçu au moins une ordonnance pour un médicament pouvant servir au soulagement de la douleur. Ainsi, au cours de 2003, la RAMQ a remboursé près de 8,5 millions d'ordonnances pour des médicaments analgésiques et antipyrétiques pour un montant total de plus de 15,5 millions de dollars (Régie de l'assurance maladie du Québec. Statistiques annuelles, 2003). Bien entendu, les coûts de la médication utilisée pour le soulagement de la douleur pour l'ensemble 
du Québec sont encore plus importants. Aux montants remboursés par la RAMQ, il faut ajouter le coût des médicaments prescrits à des personnes qui ne sont pas couvertes par le programme d'assurance médicament provincial, le coût des médicaments reçus à l'hôpital et le coût des médicaments qui ont été obtenus sans ordonnance. D'ailleurs, on rapporte que l'automédication ou le recours à des médicaments non prescrits constitue une part importante des médicaments utilisés pour le traitement de la douleur (Phillips, 2003).

Le caractère chronique de la douleur est une composante déterminante de l'importance des coûts qui lui sont reliés. Ainsi, par opposition aux coûts associés au traitement des douleurs lombaires ou des douleurs neuropathiques par exemple, qui sont généralement des douleurs chroniques, on rapporte un coût beaucoup plus faible pour la médication utilisée pour le soulagement de la douleur aiguë postopératoire (Dalton et al., 2000).

En outre, l'utilisation des médicaments pour la douleur n'est pas sans conséquences négatives. Par exemple, les anti-inflammatoires non stéroidiens, fréquemment utilisés pour le soulagement de la douleur, sont associés entre autres au développement de complications gastro-intestinales tel l'ulcère duodénal. Naturellement, ces complications entraînent, elles aussi, des coûts de santé non négligeables (Goldberg et al., 2003).

Il n'existe pas d'estimation précise du coût des services médicaux (visites chez le médecin, visites à l'urgence ou aux cliniques externes des hôpitaux et hospitalisation) sollicités pour cause de douleur. Par contre, étant donné l'importance du nombre de personnes souffrant de douleur, et parce que ce problème constitue une des principales causes de visites médicales, il est clair qu'à ce chapitre aussi la douleur est associée à des coûts importants.

Ainsi, bien que difficilement quantifiable globalement, la douleur constitue un fardeau économique important pour les personnes qui en souffrent, pour les employeurs, pour le système de soins de santé et pour la société en général. Le coût des interventions visant à réduire la douleur est important et est en grande partie, au Canada, supporté par nos régimes publics de soins de santé. Toutefois, il est essentiel de mettre les coûts associés au soulagement de la douleur en opposition au fardeau économique que représente la douleur elle-même. Ainsi, l'adoption d'interventions permettant un soulagement adéquat de la douleur pourrait se solder par des gains de productivité dont bénéficiera l'ensemble de la société.

\section{Bibliographie}

ABERNETHY, A.P., G.P. SAMSA et D.B. MATCHAR (2003). "A clinical decision and economic analysis model of cancer pain management », dans Am. J. Manag. Care, octobre, 9(10), p. 651-664.

BERGER, A, E.M. DUKES et G. OSTER (2004). "Clinical characteristics and economic costs of patients with painful neuropathic disorders», dans J Pain, avril, 5(3), p. 143-149.

DALTON, J.A., J. CARLSON, C. LINDLEY, W. BLAU, R. YOUNGBLOOD et S.M.GREER (2000). «Clinical economics, calculating the cost of acute postoperative pain medication ", dans J. Pain. Symptom. Manag., avril, 19(4), p. 295-308.

GOLDBERG, G.A., S.S. KIM, R. SEIFELDIN, M.P. PATTON et M. HABERMAN (2003). «Health care costs associated with suboptimal management of persistent pain ", dans Manag. Care, août, 12(8 Suppl. Improving pain), p. 14-17.

HUTUBESSY, R.C., M.W. VAN TULDER, H. VONDELING et L.M. BOUTER (1999). "Indirect costs of back pain in the Netherlands: a comparison of the human capital method with the friction cost méthode», dans Pain, mars, 80(1-2), p. 201-207.

JOISH, V.N. et D.I. BRIXNER (2002). «Back pain and productivity: measuring worker productivity from an employer's perspective», dans J. Pain Palliat. Care Pharmacother., 18(2), p. $79-85$.

KATZ, W.A. (2002). «Musculoskeletapain and its socioeconomic implications», dans Clin. Rheumatol., février, 21 Suppl. 1, p. S2-S4.

KOOPMANSCHAP, M.A., F.F. RUTTEN, B.M. VAN INEVELD et L. VAN ROIJEN (1995). " The friction cost method for measuring indirect costs of disease ", dans J. Health Econ., juin, 14(2), p. 171-89.

MAETZEL, A. et L. LI (2002). «The economic burden of low back pain: a review of studies published between 1996 and 2001 », dans Best Pract. Res. Clin. Rheumatol., janvier, 16(1), p. 23-30.

MANIADAKIS, N. et A. GRAY (2000). «The economic burden of back pain in the UK», dans Pain, janvier, 84(1), p. 95-103.

MAPEL D.W., M. SHAINLINE, K. PAEZ et M. GUNTER (2004).«Hospital, pharmacy, and outpatient costs for osteoarthritis and chronic back pain ", dans J. Rheumatol., mars, 31(3), p. 573-583.

PAI, S. et L.J. SUNDARAM (2004).«Low back pain: an economic assessment in the United States ", dans Orthop. Clin. North Am. janvier, 35(1), p. 1-5.

PHILLIPS, C.J. (2003). «Prise en charge de la douleur, réflexions sur l'économie de la santé et la qualité de vie», dans Drugs, 63(2).

PHILLIPS, C.J. (2001). «The real cost of pain management», dans Anaesthesia, novembre, 56(11), p. 1031-1033.

RÉGIE DE L'ASSURANCE MALADIE DU QUÉBEC (2003). Statistiques annuelles.
ROTHERMICH E.A. et D.S. PATHAK (1999). «Productivity-cost controversies in cost-effectiveness analysis: review and research agenda», dans Clin. Ther., janvier, 21(1), p. 255-267.

SENNFALT. K., P. CARLSSON, G. SANDBLOM et E.VARENHORST (2004). "The estimated economic value of the welfare loss due to prostate cancer pain in a defined population», Acta Oncol., 43(3), p. 290-296.

STEWART, W.F, J.A.RICCI，E. CHEE, D. MORGANSTEIN et R. LIPTON (2003). «Lost productive time and cost due to common pain conditions in the US workforce», dans JAMA, 12 novembre, 290(18), p. 2443-2454.

STRAUS, B.N. (2002). "Chronic pain of spinal origin: the costs of intervention », dans Spine, 15 novembre, 27(22), p. 2614-2619, discussion p. 2620.

WILDENHAUS, K.J. (2004). "Feeling the economic effects of back pain", dans AHIP Cover, mai-juin, 45(3), p. 78-80, 82, 84. 